Bull. Korean Math. Soc. 48 (2011), No. 2, pp. 335-342

DOI 10.4134/BKMS.2011.48.2.335

\title{
SPHERE-FOLIATED MINIMAL AND CONSTANT MEAN CURVATURE HYPERSURFACES IN PRODUCT SPACES
}

\author{
Keomkyo Seo
}

\begin{abstract}
In this paper, we prove that minimal hypersurfaces when $n \geq 3$ and nonzero constant mean curvature hypersurfaces when $n \geq 2$ foliated by spheres in parallel horizontal hyperplanes in $\mathbb{H}^{n} \times \mathbb{R}$ must be rotationally symmetric.
\end{abstract}

\section{Introduction}

In $\mathbb{R}^{3}$, catenoids and Riemann's examples are the only minimal surfaces foliated by circles. However, in higher-dimensional Euclidean space, there are no examples of non-rotationally symmetric minimal hypersurfaces such as Riemann's examples in $\mathbb{R}^{3}$. In 1991, Jagy [6] proved that if $M^{n}$ is a minimal hypersurface in $\mathbb{R}^{n+1}(n \geq 3)$ and foliated by $(n-1)$-dimensional spheres in parallel hyperplanes, then $M^{n}$ is rotationally symmetric about the axis containing the centers of all the spheres. This result has been generalized to other spaces forms: sphere, the hyperbolic and Lorentz-Minkowski space (See [7], [8], and [11]).

In $\mathbb{H}^{2} \times \mathbb{R}$, Nelli and Rosenberg [9] found a rotationally symmetric minimal surface which is called a catenoid in $\mathbb{H}^{2} \times \mathbb{R}$. In [4], Hauswirth provided several examples of minimal surfaces foliated by horizontal curves of constant curvature in $\mathbb{H}^{2} \times \mathbb{R}$. In particular, he constructed a two-parameter family of Riemann type surfaces. Recently, Bérard and Sa Earp [2] obtained some results on total curvature and index of higher-dimensional catenoids in $\mathbb{H}^{n} \times \mathbb{R}$. On the other hand, Nelli et al. [10] described the geometric behavior of rotationally symmetric constant mean curvature surfaces in $\mathbb{H}^{2} \times \mathbb{R}$. They showed that for $|H|>1 / 2$, the properties of rotationally symmetric constant mean curvature surfaces in $\mathbb{H}^{2} \times \mathbb{R}$ are analogous to those of the Delaunay surfaces in $\mathbb{R}^{3}$.

Received July 21, 2009; Revised September 9, 2009.

2010 Mathematics Subject Classification. 53A10, 53C42.

Key words and phrases. foliation, constant mean curvature, rotationally symmetric hypersurface, product space.

This research was supported by the Sookmyung Women's University Research Grants 2010

(C)2011 The Korean Mathematical Society 
Rotationally symmetric constant mean curvature surfaces in $\mathbb{H}^{2} \times \mathbb{R}$ have been studied in $[1,3,5,12]$.

Throughout this paper, we consider the upper half-space model of hyperbolic space

$$
\mathbb{H}^{n}=\left\{\left(x_{1}, \ldots, x_{n}\right) \in \mathbb{R}^{n}: x_{n}>0\right\}
$$

equipped with the metric $d s^{2}=\frac{\left(d x_{1}\right)^{2}+\cdots+\left(d x_{n}\right)^{2}}{x_{n}{ }^{2}}$. For a product space $\mathbb{H}^{n} \times \mathbb{R}$, we fix the metric $d s^{2}+\varepsilon d t^{2}(\varepsilon= \pm 1)$. This metric is called Riemannian if $\varepsilon=1$ and Lorentzian if $\varepsilon=-1$.

In this paper, we study hypersurfaces foliated by $(n-1)$-dimensional spheres lying in parallel hyperplanes in some Riemannian and Lorentzian product spaces. In Section 2, we shall prove that minimal hypersurfaces $(n \geq 3)$ and non-zero constant mean curvature hypersurfaces $(n \geq 2)$ foliated by $(n-1)$ dimensional spheres in parallel horizontal hyperplanes in the Riemannian product $\mathbb{H}^{n} \times \mathbb{R}$ should be rotationally symmetric (Theorem 2.1 ). As a consequence, one can see that there is no Riemann type minimal hypersurfaces foliated by $(n-1)$-dimensional spheres in $\mathbb{H}^{n} \times \mathbb{R}$ for $n \geq 3$. We shall use Jagy's idea [6] to prove this result (See also [8]). A key ingredient of the proof is the following. We describe a hypersurface $M$ in $\mathbb{H}^{n} \times \mathbb{R}$ locally as the level set for a smooth function $f$. If we orient $M$ by the unit normal vector field $N=-\frac{\nabla f}{|\nabla f|}$, then the mean curvature $H$ is given by

$$
n H=-\operatorname{div} \frac{\nabla f}{|\nabla f|},
$$

where $\nabla$ and div denote the gradient and divergence in $\mathbb{H}^{n} \times \mathbb{R}$, respectively. A straightforward computation using the fact that $M$ is foliated by spheres in parallel horizontal hyperplanes gives us the conclusion. In Section 3, applying the similar arguments as in Section 2, we prove an analogue in the Lorentzian product $\mathbb{H}^{n} \times \mathbb{R}$.

The author would like to thank the referee for useful suggestions on improving the presentation of this paper.

\section{Sphere-foliated hypersurfaces in the Riemannian product $\mathbb{H}^{n} \times \mathbb{R}$}

In $\mathbb{H}^{n} \times \mathbb{R}$, a one-parameter family of hyperplanes $\mathbb{H}^{n} \times\{t\}$ for $t \in \mathbb{R}$ are called parallel horizontal hyperplanes. We will deal with hypersurfaces foliated by spheres in parallel horizontal hyperplanes in the Riemannian product space $\mathbb{H}^{n} \times \mathbb{R}$.

Theorem 2.1. Let $M$ be an $n$-dimensional hypersurface with constant mean curvature $H$ in the Riemannian product $\mathbb{H}^{n} \times \mathbb{R}$ and foliated by spheres in parallel horizontal hyperplanes. If $H \neq 0$ or $H=0$ and $n \geq 3$, then $M$ is a rotationally symmetric hypersurface.

Before proving the above theorem, we need the following well-known fact. 
Lemma 2.2 ([13], pp. 81-82). If an $(n-1)$-dimensional sphere has Euclidean center $(0, \ldots, 0, k) \in \mathbb{R}_{+}^{n}:=\left\{\left(x_{1}, \ldots, x_{n}\right): x_{n}>0\right\}$ and a Euclidean radius $r$, then it has the hyperbolic center $(0, \ldots, 0, K) \in \mathbb{R}_{+}^{n}$ and the hyperbolic radius $R$, where

$$
K=\sqrt{k^{2}-r^{2}} \text { and } R=\frac{1}{2} \ln \frac{k+r}{k-r} .
$$

Proof of Theorem 2.1. Let $P_{t_{1}}=\mathbb{H}^{n} \times\left\{t_{1}\right\}$ and $P_{t_{2}}=\mathbb{H}^{n} \times\left\{t_{2}\right\}$ be two horizontal hyperplanes of the foliation for $t_{1}<t_{2}$. Let $M^{*}$ be the piece of $M$ between $P_{t_{1}}$ and $P_{t_{2}}$. The boundary $\partial M^{*}$ of $M^{*}$ consists of two $(n-1)$-dimensional spheres $\left(M^{*} \cap P_{t_{1}}\right) \cup\left(M^{*} \cap P_{t_{2}}\right)$. After an isometric transformation in $\mathbb{H}^{n} \times \mathbb{R}$, we may assume that the hyperbolic centers of the two boundary spheres are given by $\left(0, \ldots, 0, k_{1}, t_{1}\right)$ and $\left(0, \ldots, 0, k_{2}, t_{2}\right)$ in $\mathbb{R}_{+}^{n} \times \mathbb{R}$ for some constants $k_{1}, k_{2}>0$, respectively. Note that these two boundary spheres are symmetric to the hyperplanes $\left\{x_{1}=0\right\}, \ldots,\left\{x_{n-1}=0\right\}$. The well-known Aleksandrov reflection principle shows that $M^{*}$ inherits the symmetries of its boundary $\partial M^{*}$. Therefore, for each $t_{1} \leq t \leq t_{2}$, the hyperbolic center of each level $M \cap\left\{x_{n+1}=t\right\}$ is symmetric to the hyperplanes $\left\{x_{1}=0\right\}, \ldots,\left\{x_{n-1}=0\right\}$. Hence it follows that the hyperbolic center of each level lies in the 2-plane $\left\{x_{1}=\cdots=x_{n-1}=0\right\}$.

Using Lemma 2.2, we parametrize the hyperbolic centers of the $(n-1)$ dimensional spheres by $t \longmapsto(0, \ldots, 0, K(t), t) \in \mathbb{H}^{n} \times \mathbb{R}$ for $t \in\left[t_{1}, t_{2}\right]$, and hence the Euclidean centers of the spheres by $t \longmapsto(0, \ldots, 0, k(t), t) \in \mathbb{H}^{n} \times \mathbb{R}$. Then it follows from Lemma 2.2 that

$$
K(t)=\sqrt{k(t)^{2}-r(t)^{2}},
$$

where $r(t)$ is the Euclidean radius of $M \cap \mathbb{H}^{n} \times\{t\}$. Note that $K(t)>0$ for $t \in\left[t_{1}, t_{2}\right]$. Moreover, we see that $M^{*}$ is the level set $\{f=0\}$ of a function $f$ given by

$$
f\left(x_{1}, \ldots, x_{n}, t\right)=\sum_{i=1}^{n-1} x_{i}^{2}+\left(x_{n}-k(t)\right)^{2}-r(t)^{2} .
$$

To prove the theorem, it is sufficient to show that

$$
\frac{d K(t)}{d t}=0
$$

which means that $M$ is a rotationally symmetric hypersurface whose rotation axis is the geodesic $\gamma(t)=\{(0, \ldots, 0, K, t)\}$ for some constant $K$. Note that the metric on $\mathbb{H}^{n} \times \mathbb{R}$ is given by

$$
\sum_{i, j} g_{i j} d x_{i} \otimes d x_{j}=\frac{1}{x_{n}^{2}} d x_{1}^{2}+\cdots+\frac{1}{x_{n}^{2}} d x_{n}^{2}+d t^{2}
$$

Since

$$
\nabla f=\sum_{i, j} g^{i j} \frac{\partial f}{\partial x_{i}} \frac{\partial}{\partial x_{j}}
$$




$$
=\left(2 x_{n}^{2} x_{1}, \ldots, 2 x_{n}^{2} x_{n-1}, 2 x_{n}^{2}\left(x_{n}-k\right),-2\left(x_{n}-k\right) k^{\prime}-2 r r^{\prime}\right),
$$

we have

$$
|\nabla f|^{2}=4\left(x_{n}^{2} x_{1}^{2}+\cdots+x_{n}^{2} x_{n-1}^{2}+x_{n}^{2}\left(x_{n}-k\right)^{2}+\left(\left(x_{n}-k\right) k^{\prime}+r r^{\prime}\right)^{2}\right) .
$$

Now we compute the mean curvature of $M^{*}$ using the equation (1.1).

$$
\begin{aligned}
-n H=\operatorname{div} \frac{\nabla f}{|\nabla f|} & =\sum_{i, j} \frac{1}{\sqrt{g}} \frac{\partial}{\partial x_{j}}\left(\sqrt{g} \frac{g^{i j}}{|\nabla f|} \frac{\partial f}{\partial x_{i}}\right) \\
& =\sum_{j} \frac{\partial Z^{j}}{\partial x_{j}}+\sum_{j} \frac{1}{\sqrt{g}}\left(\frac{\partial \sqrt{g}}{\partial x_{j}}\right) Z^{j},
\end{aligned}
$$

where $Z^{j}=\sum_{i} \frac{g^{i j}}{|\nabla f|} \frac{\partial f}{\partial x_{i}}$ and $g=\operatorname{det}\left(g_{i j}\right)=x_{n}^{-2 n}$. Then we have

$$
\begin{aligned}
-n H= & \frac{x_{n}^{2}}{S}-\frac{\left(x_{n}^{2} x_{1}\right)^{2}}{S^{3}}+\cdots+\frac{x_{n}^{2}}{S}-\frac{\left(x_{n}^{2} x_{n-1}\right)^{2}}{S^{3}} \\
& +\frac{x_{n}^{2}+2 x_{n}\left(x_{n}-k\right)}{S}-\frac{x_{n}^{2}\left(x_{n}-k\right)\left\{x_{n} r^{2}+x_{n}^{2}\left(x_{n}-k\right)+A k^{\prime}\right\}}{S^{3}} \\
& +\frac{B}{S}-\frac{A\left\{x_{n}^{2}\left(x_{n}-k\right) k^{\prime}+A B\right\}}{S^{3}} \\
& +x_{n}^{n}\left(-n x_{n}^{-n-1}\right) \frac{x_{n}^{2}\left(x_{n}-k\right)}{S},
\end{aligned}
$$

where $A=\left(x_{n}-k\right) k^{\prime}+r r^{\prime}, B=k^{\prime 2}-\left(x_{n}-k\right) k^{\prime \prime}-r^{\prime 2}-r r^{\prime \prime}$, and

$$
\begin{aligned}
S=\frac{|\nabla f|}{2} & =\sqrt{x_{n}^{2} x_{1}^{2}+\cdots+x_{n}^{2} x_{n-1}^{2}+x_{n}^{2}\left(x_{n}-k\right)^{2}+\left(\left(x_{n}-k\right) k^{\prime}+r r^{\prime}\right)^{2}} \\
& =\sqrt{x_{n}^{2} r^{2}+A^{2}} .
\end{aligned}
$$

Thus we have

$$
\begin{aligned}
-n H S^{3}= & 2 A^{2} x_{n}^{2}+(n-1) k r^{2} x_{n}^{3}+(n-2) k x_{n} A^{2} \\
& +r^{2} x_{n}^{2} B-2 x_{n}^{3} k^{\prime} A+2 k k^{\prime} x_{n}^{2} A .
\end{aligned}
$$

Squaring the above equation, we obtain

$$
\begin{aligned}
n^{2} H^{2} S^{6}= & n^{2} H^{2}\left\{x_{n}^{2} r^{2}+\left(k^{\prime} x_{n}+r r^{\prime}-k k^{\prime}\right)^{2}\right\}^{3} \\
(2.2)= & {\left[\left\{2 r r^{\prime} k^{\prime}+(n-2) k k^{\prime 2}+(n-1) k r^{2}-r^{2} k^{\prime \prime}\right\} x_{n}^{3}\right.} \\
& +\left\{\left(k^{\prime 2}+r^{\prime 2}-r r^{\prime \prime}+k k^{\prime \prime}\right) r^{2}+2(n-3) k k^{\prime} r r^{\prime}-2(n-2) k^{\prime 2} k^{2}\right\} x_{n}^{2} \\
& \left.+k(n-2)\left(r r^{\prime}-k k^{\prime}\right)^{2} x_{n}\right]^{2} .
\end{aligned}
$$

Suppose that $H \neq 0$. Let us fix a section $t$. Since $x_{n}$ is varied, we regard (2.2) as an equation on $x_{n}$ where the coefficients are functions of the independent variable $t$. Comparing the degree 0 -terms in both sides of (2.2), we get

$$
n^{2} H^{2}\left(r r^{\prime}-k k^{\prime}\right)^{6}=0 .
$$


Therefore it follows that

$$
\frac{d K(t)}{d t}=\frac{d}{d t} \sqrt{k(t)^{2}-r(t)^{2}}=\frac{k k^{\prime}-r r^{\prime}}{\sqrt{k^{2}-r^{2}}}=0 .
$$

Now suppose that $H=0$ and $n \geq 3$. Comparing the coefficients of the degree 2-terms in both sides of (2.2), we have

$$
k(n-2)\left(r r^{\prime}-k k^{\prime}\right)^{2}=0 .
$$

Therefore $r r^{\prime}-k k^{\prime}=0$, which also implies that

$$
\frac{d K(t)}{d t}=0
$$

Hence we can conclude that $M$ is a rotationally symmetric hypersurface in both cases.

Remark. In $\mathbb{H}^{2} \times \mathbb{R}$, Hauswirth [4] constructed several Riemann type minimal surfaces foliated by circles. However, as mentioned in the introduction, it follows from the above theorem that there is no Riemann type minimal hypersurface which is not rotationally symmetric and foliated by $(n-1)$-dimensional spheres lying in parallel horizontal hyperplanes in $\mathbb{H}^{n} \times \mathbb{R}$ when $n \geq 3$.

\section{Sphere-foliated hypersurfaces in the Lorentzian product $\mathbb{H}^{n} \times \mathbb{R}$}

An immersed hypersurface $M$ in the Lorentz product space $\mathbb{H}^{n} \times \mathbb{R}$ endowed with the Lorentzian metric

$$
d s^{2}=\frac{\left(d x_{1}\right)^{2}+\cdots+\left(d x_{n}\right)^{2}}{x_{n}{ }^{2}}-(d t)^{2}
$$

is called spacelike if the induced metric on $M$ is a Riemannian metric. If the hypersurface is locally the level set of a smooth function $f$, the fact that $M$ is spacelike means that $\nabla f$ is a timelike vector:

$$
\langle\nabla f, \nabla f\rangle<0 \text {. }
$$

If we orient $M$ by the unit normal vector field $N=-\frac{\nabla f}{|\nabla f|}$, then the mean curvature $H$ is given by

$$
n H=-\operatorname{div} \frac{\nabla f}{|\nabla f|},
$$

where $|\nabla f|=\sqrt{-\langle\nabla f, \nabla f\rangle}$ and div denotes the divergence with respect to the Lorentzian metric on the product space $\mathbb{H}^{n} \times \mathbb{R}$.

As in the proof of Theorem 2.1, consider two horizontal hyperplanes of the foliation $P_{t_{1}}=\mathbb{H}^{n} \times\left\{t_{1}\right\}$ and $P_{t_{2}}=\mathbb{H}^{n} \times\left\{t_{2}\right\}$ for $t_{1}<t_{2}$. Applying the Aleksandrov reflection principle in $\mathbb{H}^{n} \times \mathbb{R}$, we see that the piece $M^{*}$ between $P_{t_{1}}$ and $P_{t_{2}}$ has the symmetries of its boundary $\partial M^{*}=\left(M^{*} \cap P_{t_{1}}\right) \cup\left(M^{*} \cap P_{t_{2}}\right)$. Therefore, for each $t_{1} \leq t \leq t_{2}$, the hyperbolic center of each level $M \cap\left\{x_{n+1}=\right.$ 
t\} lies in the same 2-plane. After a translation in $\mathbb{H}^{n} \times \mathbb{R}$, we may assume that this 2-plane is defined by $x_{1}=\cdots=x_{n-1}=0$.

Using Lemma 2.2 again, we can parametrize the hyperbolic centers of the $(n-1)$-dimensional spheres by $t \longmapsto(0, \ldots, 0, K(t), t) \in \mathbb{H}^{n} \times \mathbb{R}$ for $t \in\left[t_{1}, t_{2}\right]$, and the Euclidean centers of the spheres by $t \longmapsto(0, \ldots, 0, k(t), t) \in \mathbb{H}^{n} \times \mathbb{R}$, where $K(t)=\sqrt{k(t)^{2}-r(t)^{2}}$ and $r(t)$ is the Euclidean radius of $M \cap \mathbb{H}^{n} \times\{t\}$. Then $M^{*}$ is the level set $\{f=0\}$ of a function $f$ defined as in (2.1). Note that the metric on the Lorentzian product $\mathbb{H}^{n} \times \mathbb{R}$ is given by

$$
\sum_{i, j} g_{i j} d x_{i} \otimes d x_{j}=\frac{1}{x_{n}^{2}} d x_{1}^{2}+\cdots+\frac{1}{x_{n}^{2}} d x_{n}^{2}-d t^{2} .
$$

Since

$$
\begin{aligned}
\nabla f & =\sum_{i, j} g^{i j} \frac{\partial f}{\partial x_{i}} \frac{\partial}{\partial x_{j}} \\
& =\left(2 x_{n}^{2} x_{1}, \ldots, 2 x_{n}^{2} x_{n-1}, 2 x_{n}^{2}\left(x_{n}-k\right), 2\left(\left(x_{n}-k\right) k^{\prime}+r r^{\prime}\right)\right)
\end{aligned}
$$

we get

$-\langle\nabla f, \nabla f\rangle=4\left(-x_{n}^{2} x_{1}^{2}-\cdots-x_{n}^{2} x_{n-1}^{2}-x_{n}^{2}\left(x_{n}-k\right)^{2}+\left(\left(x_{n}-k\right) k^{\prime}+r r^{\prime}\right)^{2}\right)$.

Using the mean curvature equation (3.1), we have

$$
-n H=\operatorname{div} \frac{\nabla f}{|\nabla f|}=\sum_{i, j} \frac{1}{\sqrt{|g|}} \frac{\partial}{\partial x_{j}}\left(\sqrt{|g|} \frac{g^{i j}}{|\nabla f|} \frac{\partial f}{\partial x_{i}}\right),
$$

where $|\nabla f|=\sqrt{-\langle\nabla f, \nabla f\rangle}$ and $|g|=\left|\operatorname{det}\left(g_{i j}\right)\right|=x_{n}^{-2 n}$.

A similar computation as in the proof of Theorem 2.1 shows that

$$
\begin{aligned}
& n^{2} H^{2}\left\{-x_{n}^{2} r^{2}+\left(k^{\prime} x_{n}+r r^{\prime}-k k^{\prime}\right)^{2}\right\}^{3} \\
= & {\left[\left\{2 r r^{\prime} k^{\prime}-(n-2) k k^{2}-(n-1) k r^{2}+r^{2} k^{\prime \prime}\right\} x_{n}^{3}\right.} \\
& +\left\{\left(k^{\prime 2}+r^{\prime 2}-r r^{\prime \prime}+k k^{\prime \prime}\right) r^{2}-2(n-1) k k^{\prime} r r^{\prime}+2(n-2) k^{\prime 2} k^{2}\right\} x_{n}^{2} \\
& \left.-k(n-2)\left(r r^{\prime}-k k^{\prime}\right)^{2} x_{n}\right]^{2} .
\end{aligned}
$$

Suppose that $H \neq 0$. Comparing the degree 0-terms in both sides of the equation (3.2) of variable $x_{n}$, we obtain

$$
n^{2} H^{2}\left(r r^{\prime}-k k^{\prime}\right)^{6}=0 .
$$

Therefore it follows that

$$
\frac{d K(t)}{d t}=\frac{d}{d t} \sqrt{k(t)^{2}-r(t)^{2}}=\frac{k k^{\prime}-r r^{\prime}}{\sqrt{k^{2}-r^{2}}}=0 .
$$


Now suppose that $H=0$ and $n \geq 3$. Comparing the coefficients of the degree 2 -terms in both sides of $(3.2)$, we have

$$
k(n-2)\left(r r^{\prime}-k k^{\prime}\right)^{2}=0 .
$$

So we have $r r^{\prime}-k k^{\prime}=0$, which also implies that

$$
\frac{d K(t)}{d t}=0
$$

From (3.3) and (3.4), it follows that the hyperbolic center of each hypersphere in parallel horizontal hyperplane lies in a vertical geodesic line of the Lorentzian product $\mathbb{H}^{n} \times \mathbb{R}$. Therefore we obtain the following.

Theorem 3.1. Let $M$ be an $n$-dimensional spacelike hypersurface with constant mean curvature $H$ in the Lorentzian product $\mathbb{H}^{n} \times \mathbb{R}$ and foliated by spheres in parallel horizontal hyperplanes. If $H \neq 0$ or $H=0$ and $n \geq 3$, then $M$ is rotationally symmetric.

\section{References}

[1] U. Abresch and H. Rosenberg, A Hopf differential for constant mean curvature surfaces in $S^{2} \times R$ and $H^{2} \times R$, Acta Math. 193 (2004), no. 2, 141-174.

[2] P. Bérard and R. Sa Earp, Minimal hypersurfaces in $\mathbb{H}^{n} \times \mathbb{R}$, total curvature and index, arXiv: $0808.3838 \mathrm{v} 1$.

[3] M. Cavalcante and J. de Lira, Examples and structure of CMC surfaces in some Riemannian and Lorentzian homogeneous spaces, Michigan Math. J. 55 (2007), no. 1, $163-181$.

[4] L. Hauswirth, Minimal surfaces of Riemann type in three-dimensional product manifolds, Pacific J. Math. 224 (2006), no. 1, 91-117.

[5] W.-T. Hsiang and W.-Y. Hsiang, On the uniqueness of isoperimetric solutions and imbedded soap bubbles in noncompact symmetric spaces. I, Invent. Math. 98 (1989), no. $1,39-58$.

[6] W. Jagy, Minimal hypersurfaces foliated by spheres, Michigan Math. J. 38 (1991), no. $2,255-270$.

[7] W. Jagy, Sphere-foliated constant mean curvature submanifolds, Rocky Mountain J. Math. 28 (1998), no. 3, 983-1015.

[8] R. López, Constant mean curvature hypersurfaces foliated by spheres, Differential Geom. Appl. 11 (1999), no. 3, 245-256.

[9] B. Nelli and H. Rosenberg, Minimal surfaces in $\mathbb{H}^{2} \times \mathbb{R}$, Bull. Braz. Math. Soc. (N.S.) 33 (2002), no. 2, 263-292.

[10] B. Nelli, R. Sa Earp, W. Santos, and E. Toubiana, Uniqueness of $H$-surfaces in $\mathbb{H}^{2} \times$ $\mathbb{R},|H| \leq 1 / 2$, with boundary one or two parallel horizontal circles, Ann. Global Anal. Geom. 33 (2008), no. 4, 307-321.

[11] S.-H. Park, Sphere-foliated minimal and constant mean curvature hypersurfaces in space forms and Lorentz-Minkowski space, Rocky Mountain J. Math. 32 (2002), no. 3, 10191044.

[12] R. Sa Earp and E. Toubiana, Screw motion surfaces in $\mathbb{H}^{2} \times \mathbb{R}$ and $\mathbb{S}^{2} \times \mathbb{R}$, Illinois J. Math. 49 (2005), no. 4, 1323-1362.

[13] S. Stahl, The Poincaré Half-Plane, Jones and Bartlett Publishers, Boston, MA, 1993. 
Department of Mathematics

SOOKMYUNG WOMEN'S UNIVERSITY

Seoul 140-742, Korea

E-mail address: kseo@sookmyung.ac.kr 